\title{
Nanorheology of Interfacial Water during Ice Gliding
}

\author{
L. Canale, ${ }^{1}$ J. Comtet, ${ }^{1}$ A. Niguès $\odot,{ }^{1}$ C. Cohen, ${ }^{2}$ C. Clanet, ${ }^{2}$ A. Siria, ${ }^{1, *}$ and L. Bocquet $\oplus^{1, \dagger}$ \\ ${ }^{1}$ Laboratoire de Physique de l'Ecole Normale Supérieure, ENS, Université PSL, CNRS, Sorbonne \\ Université, Université Paris-Diderot, Sorbonne Paris Cité, \\ UMR CNRS 8550, 24 rue Lhomond, F-75005 Paris, France \\ ${ }^{2}$ LadHyX, UMR CNRS 7646, Ecole Polytechnique, 91128 Palaiseau, France
}

(Received 2 July 2019; revised manuscript received 4 September 2019; published 4 November 2019)

\begin{abstract}
The slipperiness of ice is an everyday-life phenomenon, which, surprisingly, remains controversial despite a long scientific history. The very small friction measured on ice is classically attributed to the presence of a thin self-lubricating film of meltwater between the slider and the ice. But while the macroscale friction behavior of ice and snow has been widely investigated, very little is known about the interfacial water film and its mechanical properties. In this work, we develop a stroke-probe force measurement technique to uncover the microscopic mechanisms underlying ice lubrication. We simultaneously measure the shear friction of a bead on ice and quantify the in situ mechanical properties of the interfacial film, as well as its thickness, under various regimes of speed and temperature. In contrast with standard views, meltwater is found to exhibit a complex viscoelastic rheology, with a viscosity up to 2 orders of magnitude larger than pristine water. The unconventional rheology of meltwater provides a new, consistent, rationale for ice slipperiness. Hydrophobic coatings are furthermore shown to strongly reduce friction due to a surprising change in the local viscosity, providing an unexpected explanation for waxing effects in winter sports. Beyond ice friction, our results suggest new avenues towards self-healing lubricants to achieve ultralow friction.
\end{abstract}

DOI: 10.1103/PhysRevX.9.041025

Subject Areas: Fluid Dynamics, Materials Science, Soft Matter

\section{INTRODUCTION}

Ice and snow exhibit outstanding friction properties, with exceptionally low friction coefficients [1]. This unique behavior is at the root of all winter sports [2,3]; it is also a major source of risk in transportation, or, in a very different context, a key ingredient in glacier sliding $[4,5]$. The low friction of ice remains, however, highly counterintuitive and paradoxical, since, comparatively, liquid water is usually considered as a bad lubricant due to its low viscosity. In spite of more than a century of investigation, the very origin of this puzzling property is not settled yet and remains highly debated. Since the seminal work of Faraday [6], a consensus has been reached on the existence of a liquidlike layer wetting the ice surface [7-12], with a thickness varying between 1 and $100 \mathrm{~nm}$ depending on the temperature [13], although the underlying mechanism of formation remains debated $[14,15]$. Now, under sliding the

\footnotetext{
*alessandro.siria@1ps.ens.fr

†1yderic.bocquet@1ps.ens.fr
}

Published by the American Physical Society under the terms of the Creative Commons Attribution 4.0 International license. Further distribution of this work must maintain attribution to the author(s) and the published article's title, journal citation, and DOI. fate of this interfacial film remains largely unknown [16]. The pioneering works of Bowden and Hughes $[17,18]$ and later Colbeck [19] have discarded pressure-melting mechanisms in ice and snow friction and suggested frictional melting: viscous dissipation generates heat, which raises the temperature in the contact region up to the melting point, hereby creating a water lubricating film. This scenario has been further explored by numerous macroscopic tribological measurements [20-23], supported—at least partly—by theoretical frameworks $[3,16,19,24]$. However, probing the in situ properties of this interfacial film remains a formidable challenge. Indeed the meltwater film is dynamically and self-consistently generated under sliding, which makes the ice-water boundary elusive to detect. Also, standard interferometry techniques fail because of the low contrast of the interface. The few existing measurements, focusing on the meltwater film thickness, led to contradictory results, with the estimated thickness varying from 5-10 $\mu \mathrm{m}$ [25] to less than $50 \mathrm{~nm}$ [26]. Even more puzzling, recent local temperature measurements have precluded full melting of an interfacial water film under sliding, contradicting standard explanations [27]. It is not an understatement that the fundamental mechanisms for the slipperiness of ice (and snow) still remain a mystery. In this work, we propose a radically new approach, which enables us to disentangle 
the various physical ingredients at stake. We investigate simultaneously the friction of a millimetric slider on ice and the corresponding interfacial mechanical properties of the meltwater film at the nanoscale. To this end, we harvest the possibilities offered by a newly introduced force measurement apparatus $[28,29]$, to realize here a "stroke-probe" tribometer with nanoscopic sensitivity. This apparatus allows us to close the gap between nanoscale and macroscale tribometry [30], which is a prerequisite for the investigation of the ice interface under sliding.

\section{STROKE-PROBE ATOMIC FORCE MICROSCOPE (AFM) EXPERIMENTS}

The experimental setup, shown in Fig. 1(a), consists of a double-mode tuning fork atomic force microscope (AFM)

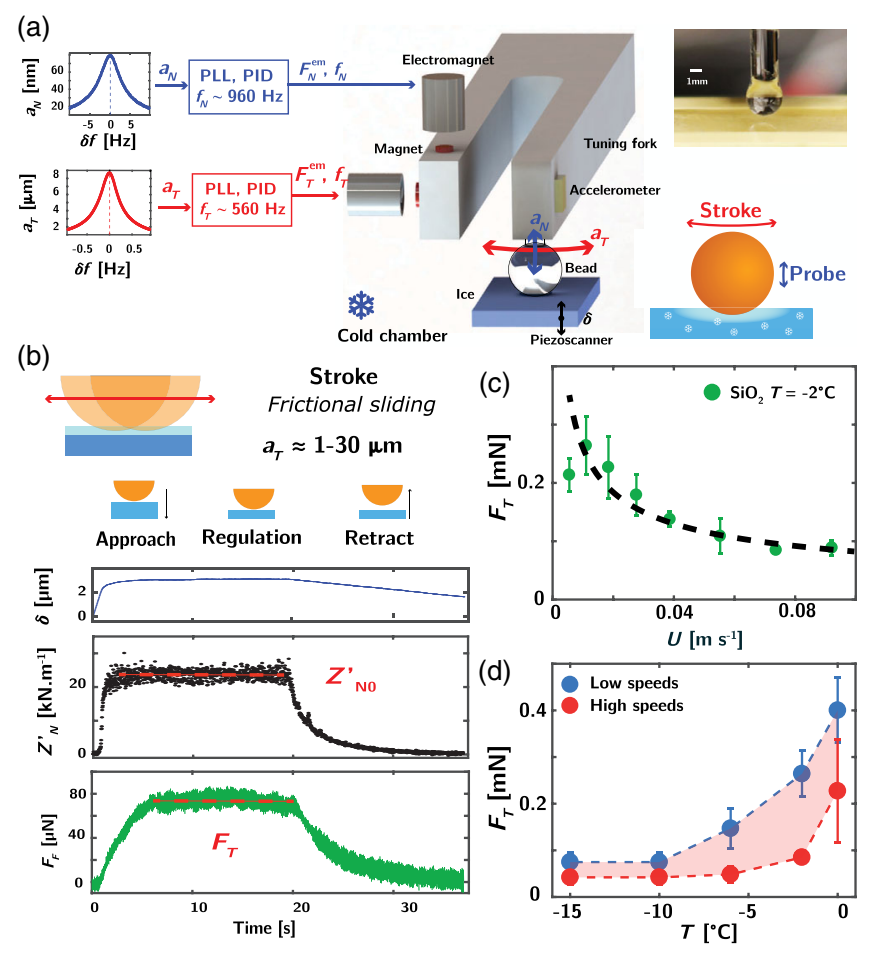

FIG. 1. Stroke-probe tribology of ice. (a) Schematic of the setup. A glass bead of radius $R \approx 1.5 \mathrm{~mm}$ is glued to a macroscopic tuning fork (not to scale). The tuning fork is excited at its resonances, leading to a tangential and normal bead oscillation in order to simultaneously shear the ice (stroke, horizontal red arrow) and measure the interfacial properties (probe, vertical blue arrow). (b) Typical approach-retract curve for the indentation distance $\delta$, the normal conservative impedance $Z_{N}^{\prime}$ and the tangential frictional force $F_{F}$ at $U=0.005 \mathrm{~m} \mathrm{~s}^{-1}$ and $T=$ $-6{ }^{\circ} \mathrm{C}$. Maintaining the contact to a prescribed normal impedance value $Z_{N 0}^{\prime}$ allows us to define a steady-state friction force $F_{T}$. (c) Steady-state friction force $F_{T}$ as a function of tangential speed $U$. The dashed line is a fit according to $F_{T} \propto U^{-\gamma}$, with $\gamma=0.5$. (d) Friction force $F_{T}$ as a function of the ice temperature for two distinct velocities $\left(U=0.01 \mathrm{~m} \mathrm{~s}^{-1}\right.$ and $\left.U=0.1 \mathrm{~m} \mathrm{~s}^{-1}\right)$, exhibiting a steady increase close to the melting point.
[28,29,31]. The setup is placed in a cold chamber with controlled temperature from $-16^{\circ} \mathrm{C}$ to $0{ }^{\circ} \mathrm{C}$ and $70 \%-80 \%$ relative humidity. A centimetric sample of ice is obtained from deionized water. Under these conditions, ice evaporation was measured to occur on a timescale much longer than the measurements. The ice temperature is directly monitored via an embedded thermocouple. A millimetric borosilicate glass bead [Fig. 1(a), see also Fig. S2 of the Supplemental Material [32] for bead characterization] is glued on one prong of a centimetric aluminium tuning fork. The system can be accurately modeled as a mass-spring resonator of large stiffness $K_{T} \approx 10^{2} \mathrm{kN} \mathrm{m}^{-1}$ and quality factor $Q_{T} \approx 2500$. An electromagnetic excitation at the tuning fork resonance frequency, $f_{T} \simeq 560 \mathrm{~Hz}$, then yields a lateral oscillatory motion of the bead parallel to the ice surface, Fig. 1(a) (red arrow). Its amplitude $a_{T}$ and phase shift $\phi_{T}$ with respect to the excitation force are monitored with an accelerometer glued on one prong. The oscillating sphere is brought into contact with the ice surface by a piezoelement with integrated position sensor of nanometric resolution. The lateral stroke of the sphere then shears the ice with an amplitude $a_{T} \sim 1-30 \mu \mathrm{m}$ and velocity $U=2 \pi a_{T} f_{T}$, typically up to $0.1 \mathrm{~m} \mathrm{~s}^{-1}$. A phaselocked loop (PLL) maintains the system at the resonance by tuning the excitation frequency $f_{T}$, and the tangential friction force $F_{F}$ is simply measured by tracking the excitation force $F_{T}^{\mathrm{em}}$ necessary to keep the oscillation amplitude $a_{T}$ constant while sliding according to $F_{F}=$ $\left(K_{T} / Q_{T}\right)\left(F_{T}^{\mathrm{em}} / F_{T, 0}^{\mathrm{em}}-1\right) \times a_{T}[29]$.

Simultaneously, we take advantage of the higher order eigenmodes of the tuning fork: as sketched in Fig. 1(a), we excite the first normal mode associated with a resonance frequency, $f_{N} \simeq 960 \mathrm{~Hz}\left(K_{N} \sim 10^{3} \mathrm{kN} \mathrm{m}^{-1}, Q_{N} \sim 200\right)$, and measure the corresponding normal force using a similar procedure as for the tangential force. This gentle probe, with a tiny normal oscillation amplitude, $a_{N} \sim 50 \mathrm{~nm}$, allows us to measure the normal mechanical impedance of the sheared ice, $Z_{N}^{*}=F_{N}^{*} / a_{N}$, with $F_{N}^{*}$ the complex normal force acting on the sphere. The real and imaginary parts of $Z_{N}^{*}=Z_{N}^{\prime}+i Z_{N}^{\prime \prime}$ correspond, respectively, to the normal conservative and dissipative mechanical impedance of the interfacial medium. We verified that the normal mode oscillation does not influence the tangential mode and the friction measurement (see Supplemental Material, Figs. S1, S6, and S9) and that the interfacial mechanical properties do not depend upon the normal oscillation amplitude (Supplemental Material, Fig. S7 [32]).

Altogether, this superposition methodology allows us to gently probe the mechanical properties of the interface while the tangential stroke shears laterally the ice surface, echoing the principle of superposition rheometry [33]. It allows performing simultaneously tribometry and rheology of the contact. Previous investigations of the ice surface using atomic force microscopy [7,34] evidenced the 
existence of the premelting water layer [7], as well as solidlike frictional properties (in conditions of low temperature and low vapor pressure) [34]. However, in comparison to these investigations using standard AFM, our experimental setup allows us to measure the lateral friction with a macroscopic probe and to have a nanoscopic sensitivity. Hence, we bridge the gap between standard AFM measurements and macroscale tribological experiments. We also emphasize that the experimental methodology and setup were validated by several control experiments on different fluids and conditions: (i) a standard silicone oil was investigated using our protocol, leading to the expected Newtonian rheology with the tabulated viscosity, see Supplemental Material Fig. S9 and Ref. [29]; (ii) ionic liquids were investigated using this protocol in a previous study, see Ref. [29] and, beyond the rheology, also evidenced molecular layering at the interface, confirming the sensitivity of the setup; (iii) the two modes (stroke-probe) methodology was successfully implemented to study the interfacial properties of liquids exhibiting complex (shear-thickening) rheology, beyond that of Newtonian fluids, see Ref. [31]. Last but not least, experiments with an alternative phase-changing material, namely solidified polyelethylene glycol 1000 (PEG-1000), were also conducted in the present study, highlighting interesting similarities with ice, which we discuss later.

Let us now describe the experimental procedure. Prior to the measurements, we first proceed to several preliminary approach-retracts of the probe under sliding, which allows leveling the surface. Then, the probe is slowly put in contact with the ice, see Fig. 1(b): the indentation distance $\delta$ increases and the friction force increases as the probe starts shearing the ice. The maximal indentation is kept small, typically $\delta_{0} \sim 3 \mu \mathrm{m}$, and this precludes dissipation due to ploughing, which has been evidenced at larger loads and indentations [35]. In the experiments, we set the normal conservative impedance $Z_{N}^{\prime}$ at a prescribed value $Z_{N 0}^{\prime}$ by adjusting the maximal indentation position $\delta_{0}$. This amounts to fixing the normal load on the sphere, which is obtained by integrating the gradient $Z_{N}^{\prime}$ over the indentation distance (see Supplemental Material, Fig. S4 [32]). This accordingly allows measuring a lateral frictional force $F_{T}$ for the prescribed load; see Fig. 1(b) (horizontal red dashed line). Afterwards, the bead is retracted slowly and the friction force decreases back smoothly to zero. Note that during the retract the normal conservative impedance does not exhibit a square root dependence with the indentation as it would be expected for a Hertzian deformation; it rather exhibits a hydrodynamic behavior, pointing to a fluidized interface. We discuss more exhaustively below the impedance's behavior. Altogether, the procedure described allows us to get reproducible measurements under a fixed load at different contact locations; see Supplemental Material, Figs. S3 and S4. Unless specifically mentioned, measurements for both frictional and rheological results presented in the main text are performed for the same value, $Z_{N 0}^{\prime}=24 \mathrm{kN} \mathrm{m}^{-1}$, corresponding to a load of $4.5 \mathrm{mN}$. The effect of the load is specifically studied in the Supplemental Material, Sec. S3.1 [32].

\section{TRIBOLOGY AND RHEOLOGY}

\section{A. Friction}

We first report in Fig. 1(c) the lateral friction force $F_{T}$ as a function of the tangential velocity $U$ (associated with $a_{T}$ in the range 1-30 $\mu \mathrm{m}$ ). The friction force does not vanish at low speeds, similarly to solid-on-solid friction. In addition, a weak power-law decay of the frictional force versus velocity is observed, with $F_{T} \propto U^{-\gamma}$ with $\gamma \sim 0.3-0.5$. We emphasize that this behavior is consistent with previous macroscopic measurements on ice (and snow) [20,21] [see also Supplemental Material, Fig. S5(b) [32] ]. Furthermore the friction force at a fixed velocity is found to be proportional to the normal load; see the Appendix Fig. 6 and Supplemental Material, S5(a) [32]. This points to a solidlike friction characterized by a friction coefficient $\mu=0.015$; this value is also in very good agreement with macroscopic measurements on ice [35]. Finally, repeating these measurements for various temperatures allows us to obtain the temperature dependence of the friction force; see Fig. 1(d). Counterintuitively, the friction force (here shown for two different velocities) is shown to increase steadily close to the melting point, echoing similar observations from previous macroscopic friction experiments [21,35]. Note that those experiments also typically exhibit a minimum in the friction force as a function of temperature (with the minimum position that varies depending on the slider material, see Refs. [23,35]). In our case, the trend at low temperatures may suggest that such a minimum for temperature would be shifted below $\sim-10^{\circ} \mathrm{C}$, although further investigation of very low temperatures (below $-16^{\circ} \mathrm{C}$ ) would be required, which is not possible with our present setup.

\section{B. Interfacial mechanics}

Now, taking advantage of the normal mode, one has access to the mechanical properties of the interfacial film under sliding. We measure both the real $\left(Z_{N}^{\prime}\right)$ and imaginary $\left(Z_{N}^{\prime \prime}\right)$ parts of the mechanical impedance, which are, respectively, related to the elastic and dissipative response of the interface [Supplemental Material, Eqs. (S.1) and (S.2)]. The variations of the normal mechanical impedance $Z_{N}^{\prime \prime}$ under contact and upon retract are shown in Fig. 2(a). We observe the same trends as for the friction force $F_{F}$ [see Fig. 1(b)]: a plateau during the regulation at $Z_{N 0}^{\prime}$ followed by a smooth decrease during the retract. Further insights into the dissipation are obtained by plotting the inverse of the dissipative impedance $1 / Z_{N}^{\prime \prime}$ as a function of the retract distance $d$; see Fig. 2(b). A first key result from this plot is that a linear variation of the inverse normal impedance is 

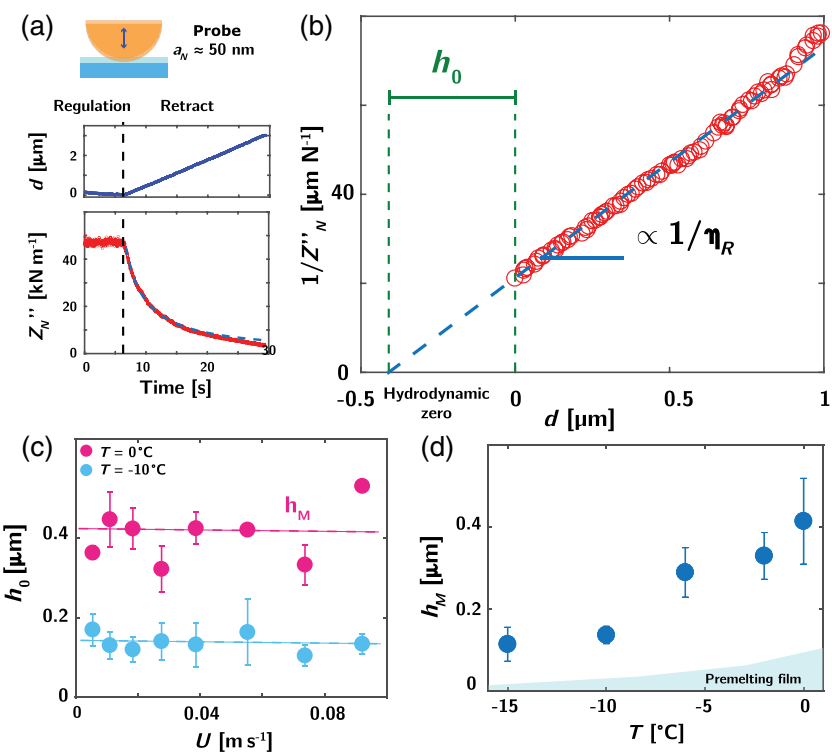

FIG. 2. Measuring the thickness of the interfacial film. (a) Retract curve for the dissipative $\left(Z_{N}^{\prime \prime}\right.$, red) normal mechanical impedance while the bead is simultaneously shearing the ice surface at speed $U=0.01 \mathrm{~m} \mathrm{~s}^{-1}\left(T=-2{ }^{\circ} \mathrm{C}\right)$. Under regulation, a steady state is reached. Upon withdrawal, the impedance $Z^{*}$ relaxes smoothly to zero. (b) Inverse of the mechanical impedance $1 / Z_{N}^{\prime \prime}$ as a function of the retract distance $d$ (during the retract phase). The linear variation of $1 / Z_{N}^{\prime \prime}$ versus the distance is accounted for by the Reynolds formula, Eq. (1), and points to the liquidlike nature of the interfacial layer. (c) Measured thickness $h_{0}$ as a function of speed for two temperatures $\left(T=-10^{\circ} \mathrm{C}\right.$ and $T=0{ }^{\circ} \mathrm{C}$ ). In each case, there is no variation with the shearing speed $U$. Measurements are performed under the same load $L=4.5 \mathrm{mN}$. (d) Average thickness $h_{M}$ as a function of $T$. As intuitively expected, we observe an increase in the film thickness as we approach the melting point. An upper limit for the thickness of the premelting film on ice taken from literature is shown in pale blue [13].

measured as a function of the retract distance $d$. Only far from the contact (large $d$ ) a small deviation from this linear behavior can be observed. This suggests a hydrodynamiclike response of the interface during the withdrawal, consistent with the Reynolds law:

$$
Z_{N}^{\prime \prime}=\frac{6 \pi \eta_{R} R^{2} \omega_{N}}{h_{\mathrm{hyd}}},
$$

where $R$ is the sphere radius, $h_{\text {hyd }}$ is the hydrodynamic film thickness, and $\eta_{R}$ the viscosity. For a nonvanishing shear velocity $U$, the interstitial fluid exhibits a viscouslike response during the retract. The observed linear behavior of $1 / Z_{N}^{\prime \prime}$ versus $d$ also indicates that the viscosity $\eta_{R}$ does not depend on the indentation depth. While a viscosity increase was evidenced for water confined below $\sim 2 \mathrm{~nm}$ [36], we note that the confinement is much less stringent here, since-anticipating from the results in the next section-the thickness of the interstitial film is always larger than hundreds of nanometers, so that no depth dependence of the viscosity is expected.

\section{Interfacial film thickness}

In the stationary state, the hydrodynamic film thickness $h_{\text {hyd }}$ is not fixed a priori but self-adjusts to reach a stationary value. According to the linear relation between $h_{\text {hyd }}$ and $1 / Z_{N}^{\prime \prime}$ highlighted above, the thickness $h_{0}$ of the stationary film can then be obtained from the measurement of the dissipative modulus $Z_{N}^{\prime \prime}$. As shown in Fig. 2(a), in the regulation regime, $Z_{N}^{\prime \prime}$ reaches a plateau as a function of time under imposed shear velocity $U$ and normal load. Accordingly, the stationary film thickness $h_{0}$ is deduced from this plateau value thanks to Eq. (1) in Fig. 2(b). On this graph, $h_{0}$ can be read by extrapolating the $1 / Z_{N}^{\prime \prime}$ line versus $h_{\text {hyd }}$ to the zero intercept (dashed blue line): $h_{0}$ then corresponds directly to the absolute value of the extrapolated hydrodynamic zero [Fig. 2(b), green dashed line]. Note that by convention, and to make the reading easier, we set in Fig. 2(b) the position of the sphere corresponding to the plateau value to $d=0$, so that $h_{0}$ corresponds directly to the absolute value of the extrapolated hydrodynamic zero. In principle, the hydrodynamic thickness is expected to be the sum of the actual film thickness and a slip length, if any. However, since ice is hydrophilic, a very small slip length is expected (typically few nanometers) [37], so that the hydrodynamic thickness should be safely identified to the real film thickness.

Repeating this procedure under various experimental conditions enables us to retrieve the stationary film thickness as a function of the lateral sliding speed, normal load, and temperature in the considered sphere-plane geometry. We report in Fig. 2(c) the variation of the interfacial film thickness $h_{0}$ with the tangential speed $U$. Surprisingly, $h_{0}$ is found to barely depend on the tangential speed, in contrast to the common belief that a larger velocity would induce a larger film $[3,19]$. Similarly, the film thickness shows a weak variation as a function of the normal load; see Fig. 6. However, the thickness increases with temperature [Fig. 2(d)], ranging from 100 to $500 \mathrm{~nm}$. The thickness of the stationary film is typically a factor of 4 higher than the values for the equilibrium premelting films [pale blue in Fig. 2(d)] [7,9-11].

\section{Rheological properties under shear}

A second fundamental lesson emerging from these measurements is that the interfacial film under shear exhibits a viscoelastic rheology, associated with a complex viscosity, $\tilde{\eta}=\eta_{R}-i \eta_{I}$ [38,39]. Indeed, as shown in Fig. 3(a), the inverse of the elastic impedance $1 / Z_{N}^{\prime}$ also exhibits a linear variation with the separation distance $d$ during the retract, allowing us to retrieve both real and imaginary parts of the viscosity, $\eta_{R}$ and $\eta_{I}$, from the corresponding slopes. In the experiments, under various 


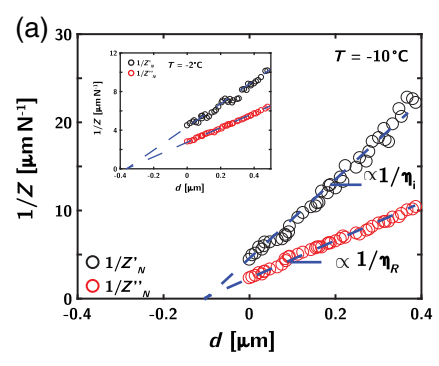

(b)

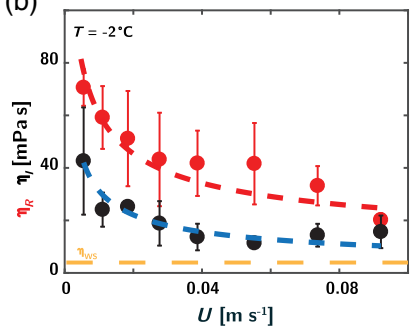

FIG. 3. Complex rheology. (a) Inverse of the mechanical impedance $1 / Z_{N}^{\prime}$ and $1 / Z_{N}^{\prime \prime}$ as a function of the retract distance $d$ for two distinct temperatures. We observe a linear behavior for both impedances, typical of a viscoelastic fluid. (b) Evolution of the real and imaginary parts of the viscosity as a function of the shearing speed $U$. The dashed lines correspond to a fit $\eta_{R, I} \propto U^{-\alpha}$ with $\alpha=0.5$. The yellow line indicates the viscosity of supercooled water $\eta_{\mathrm{WS}}$.

conditions (speed, temperature), we observe that the linear extrapolations of the inverse elastic and dissipative moduli cross at the same hydrodynamic zero within $30 \%$ error. Such a viscoelastic response of the interstitial film is analogous to that of complex fluids, e.g., polymers and polyelectrolytes [38,39]. Quantitatively, a first striking result is that the measured viscosity $\eta_{R}$ under shear is much higher than the typical viscosity of supercooled water at the same temperature; see Fig. 3(b) (orange dashed line) [40]. Both the real and the imaginary part of the viscosity follow a weak power-law decay as a function of the tangential speed, similarly to the friction force: $\eta_{R, I} \propto$ $U^{-\alpha}$ with $\alpha \sim 0.3-0.5$; see Fig. 3(b). Finally, $\eta_{R}$ is found to increase tremendously towards the melting point and reaches a value close to 2 orders of magnitude higher than water at $0^{\circ} \mathrm{C}$ [Fig. 4(a)]. On the other hand, the imaginary (elastic) part $\eta_{I}$ is found to be less sensitive to temperature, but the corresponding elastic modulus $G^{\prime}=2 \pi f_{N} \eta_{I}$, typically lies in the range of $10^{2} \mathrm{~Pa}$ [Fig. 4(b)], highlighting the strong elastic response of the film. As a side note, one may remark that the measured values for the elastic impedance
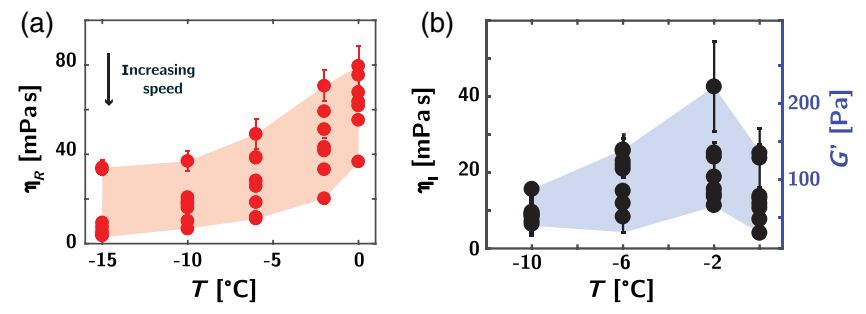

FIG. 4. Temperature effects. (a) Evolution of the real part of the viscosity (dissipation) as a function of temperature: we observe a steady increase toward the melting point, reminiscent of the friction force trend [see Fig. 1(c)]. (b) Imaginary (elastic) part of the viscosity of the interstitial fluid as a function of temperature. The right-hand axis of (b) provides the corresponding elastic modulus $G^{\prime}$.
$1 / Z_{N}^{\prime}$ in the stationary state are much larger than the estimates for a capillary contribution due to a meniscus: $\gamma R / h_{0} \sim 1 \mathrm{kN} \mathrm{m}^{-1} \ll Z_{N 0}^{\prime} \sim 20 \mathrm{kN} \mathrm{m}^{-1}$, with $\gamma$ a typical surface tension, and furthermore with opposite sign. Moreover, the estimated dissipation due to the contact line displacement and meniscus dissipation would be on the order of few $\mu \mathrm{N}$, almost 2 orders of magnitude smaller than the measured friction force. This shows that capillary effects are negligible here.

Altogether, our observations converge to an unexpected complex rheology for the meltwater. A first comment is that the interfacial water film under shear is "as viscous as an oil," with a viscosity up to 2 orders of magnitude larger than bare water. This points to an unexpected rationale for the exceptional friction properties of ice, contrasting with the bad lubricant behavior of bare liquid water. Indeed, a viscous film is a prerequisite to properly lubricate the contact: it limits squeeze-out, thereby avoiding direct solid-on-solid contact. In contrast to standard water, the "slimy melt water," which is generated under sliding, is an excellent lubricant. The complex rheology of meltwater has been completely overlooked up to now in the modeling of ice friction. The latter usually assumes bare Newtonian water and focuses on the interplay between frictional heating and the thickness of the meltwater film. Our findings suggest to reconsider the standard framework for ice friction, as well as the dissipative mechanisms occurring in the lubricating film.

\section{E. Effect of hydrophobic coatings}

Last but not least, a puzzling standard practice in winter sports is to use hydrophobic coatings to reduce friction, typically wax containing fluor additives [18]. However, adding hydrophobic coatings to favor water lubrication may seem counterintuitive, and the very origin of this behavior remains mysterious. To this end, we have investigated the friction properties of hydrophobic silanized silica beads. Here the sliders differ from the previous glass spheres only by a molecular silane layer coated on the surface of the bead. As highlighted in Fig. 5(a), the hydrophobic treatment leads to a drastic reduction of friction, as much as a factor of 10 , as compared to the standard glass surface. The friction reduction becomes stronger close to the melting point. This is in agreement with observations for the effect of wax on snow friction [18]. To get more insights into the dissipative mechanism at stake, we plot in Figs. 5(b) and 5(c) the respective film thickness and viscosity $\eta_{R}$ for the hydrophobic and hydrophilic sliders. Our measurements highlight that this friction reduction is not associated with a modified hydrodynamic film thickness $h_{0}$ [Fig. 5(b)]. On the one hand, this is somehow surprising since it excludes a priori the effect of a finite hydrodynamic slippage at the surface, which may occur for complex fluids [39,41] (slippage would indeed enhance the hydrodynamic thickness of the film according 

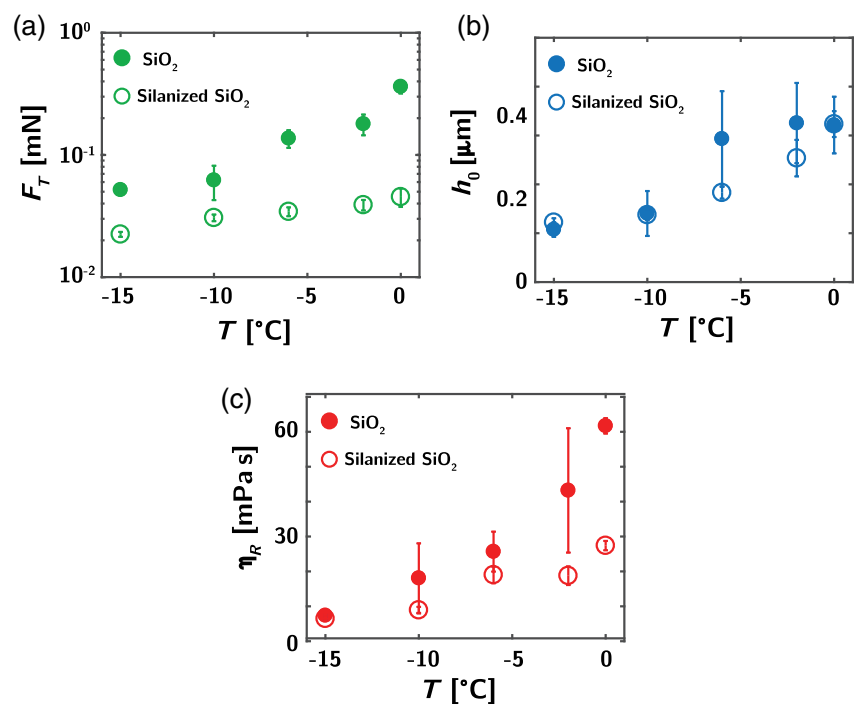

FIG. 5. Effects of the hydrophobic coatings. (a) Comparison of the friction force for a silanized sphere and bare glass for $U=$ $2.8 \mathrm{~cm} \mathrm{~s}^{-1}$ as a function of temperature. We measure a drastic reduction of friction for the hydrophobic glass sphere. (b) Comparison of the film thicknesses between the hydrophilic and hydrophobic coatings, showing similar thicknesses. (c) Comparison of the dissipative part of the viscosity: at high temperatures, the hydrophobic coating is characterized by a lower viscosity $\eta_{R}$, in line with the observed reduced friction. The effect is reduced at lower temperatures.

to $h_{0}=h_{\text {layer }}+b$, with $b$ the slip length, which is not observed). On the other hand, it confirms that the hydrodynamic thickness that we measure is not affected by a slippage effect, even in the case of bare glass. Rather, we observe a clear reduction in the real part of the viscosity for the hydrophobic glass as compared to the hydrophilic case. This effect is amplified as the temperature approaches the melting point [Fig. 5(c)]. The trend is also qualitatively the same as for the friction force. The relationship between the viscosity of the interstitial medium and the surface properties suggests that hydrophobicity may affect the buildup of the interstitial film. Altogether, these findings show that surface effects at the nanometric scale can strongly impact macroscopic ice friction. We note that in winter sports, wax coatings are not only hydrophobic, but their composition is chosen such that their hardness adjusts the ski sole to that of the snow or ice grains. Actually, snow is a much more complex material than ice: it is a porous material involving a mixture of soft snow, hard ice, and water. While our results shed some light on the hydrophobic effects, the specific interplay between elasticity, wear, and hydrodynamic properties at stake in snow has still to be completely uncovered.

\section{F. Alternative material}

Beyond ice, it is interesting to compare these results with an alternative material, which might also undergo a phase change under shear. To this end, we performed a similar study on solid polyethylene glycol 1000 (PEG 1000). This waxy material is solid at room temperature, but becomes liquid around $35^{\circ} \mathrm{C}$. We followed the very same experimental procedure as detailed above for ice, exploring its frictional and rheological response under shear at room temperature $\left(\sim 24^{\circ} \mathrm{C}\right)$. The results are reported in the Supplemental Material, Figs. S9 and S10 [32]. The lateral friction force $F_{T}$ is measured to be an affine function of the shear velocity, $F_{T}=F_{T}^{0}+\alpha U$, with a finite friction force $F_{T}^{0} \simeq 0.1 \mathrm{mN}$ as $U \rightarrow 0$ and $\alpha \simeq 0.015 \mathrm{~kg} \mathrm{~s}^{-1}$ a friction coefficient. In parallel, we investigated the rheological properties of the interface using the normal mode $\left(Z_{N}^{\prime \prime}\right.$ as a function of confinement, see Supplemental Material, Fig. S10). As for ice, these measurements highlight a viscouslike response of the interstitial material in the tested range of shear velocities, with the inverse dissipative modulus $1 / Z_{N}^{\prime \prime}$ proportional to the distance. In the present case, the viscosity is then measured to be $\eta_{R} \simeq 0.5 \mathrm{~Pa} \mathrm{~s}$, and independent of the shear velocity $U$ [Supplemental Material, Fig. S10(d)]. It is interesting to compare this value to the viscosity of PEG 1000 at the melting point. We performed standard rheometry measurements (for various temperatures above $35^{\circ} \mathrm{C}$ ) and found a value ranging from $\eta_{\text {PEG }} \simeq 0.4 \mathrm{Pas}$ close to melting down to $\eta_{\mathrm{PEG}} \simeq 0.1 \mathrm{~Pa}$ s for larger temperatures; see Supplemental Material, Fig. S11 [32]. These values are also in agreement with tabulated values. Hence, the viscosity of the interstitial material is slightly larger than the viscosity in its liquid phase. As for ice, the measurement of $Z^{\prime \prime}{ }_{N}$ also provides a value for the hydrodynamic thickness $h_{0}$ of the melted layer under shear, which is measured to be $h_{0} \approx 20 \mu \mathrm{m}$ and independent of the shear velocity [Supplemental Material, Fig. S10(c)]. As we discuss below, the similarities in behavior between ice and PEG friction should help building a common framework to rationalize the shear mechanism of "phase-changing" materials.

\section{SUMMARY AND DISCUSSION}

Overall, it is interesting to note that, in spite of the strong difference in the nature of ice and PEG 1000 as materials, some similarities in their frictional behavior do emerge: in both cases, a finite friction force is measured in the limit $\mathrm{U} \rightarrow 0$, i.e., a yielding behavior of the interstitial film with a threshold force (or stress) to induce flow; furthermore, the rheological response of the interstitial film under lateral shear exhibits a hydrodynamiclike response in the tested velocity range. Overall the film response is intermediate between that of a pure solid and a pure liquid, with a yielding behavior and shear-induced fluidization. This behavior bares similarities with solid-on-solid friction where the interstitial joint is shown to exhibit the phenomenology of soft glasses [42]. In a different context, this also echoes closely the observation for fluidization of granular materials, which is induced by an independent flow 
agitation, and results in an effective viscosity of the (otherwise yielding) material [43]. The emerging picture is in contrast with the prevailing theories for ice friction, which assumed a straight transition from a crystalline phase to the bare liquid water phase. However, it is consistent with the fact that shear cannot lead to a full liquefaction of the contact since this would require a very strong temperature increase in the contact, in contrast with various experimental observations in our experiments, see Fig. 7 , as well as in others $[21,27]$. The recent experiments of Ref. [27], reporting microscale infrared thermography and optical measurements of snow-grain contacts, actually highlighted abrasion, rather than melting, of the interstitial contact region. Gathering these experimental observations, a tempting explanation for the observed response is accordingly that, under abrasive wear, a suspension of liquid and submicron (ice or PEG) debris is formed, hence resulting in composite lubrification of the contact. Interestingly, a similar mechanism has also been recently highlighted in the context of rocks sliding [44]: the comminution of quartzite and the consequent formation of nanoparticles enables its fluidization below its melting point and facilitates slip weakening, leading to large earthquakes. Hence, this abrasion mechanism might be a general feature of phase-changing materials under shear close to their melting point. For ice and PEG, the lateral shear is expected to fluidize the (solid-liquid) mixture within the interstitial layer, again similar to Ref. [43], and the interstitial material should exhibit a complex rheology typical of dense suspensions [45]. The only difference is that here the grains constituting the suspension should self-adjust under the imposed shear, load, and temperature. For ice, the increase of viscosity with temperature may be interpreted as an increasing density of ice fragments when the ice becomes softer close to the melting point; also higher normal loads may lead to higher indentations and abrasions, providing more debris and higher viscosities. As a matter of fact, the viscosity extrapolated at vanishing load, obtained from the linear variation of the viscosity with the load, see Fig. 6(d), does perfectly match the viscosity of supercooled water at $-6^{\circ} \mathrm{C}$, suggesting again that the further rise is a direct consequence of the abrasion during contact. We leave for future works the full structural characterization of the interstitial material under shear, e.g., using spectroscopic measurements.

We finally note that, as argued by Bluhm et al., frictional melting phenomena during ice friction is not expected to be measurable in more classical AFM investigations because of the small contact sizes and low shearing speeds. This is actually in agreement with the large friction coefficient that they measure [34], although at lower temperature and lower water vapor pressure. It would therefore be desirable to extend the study using the present methodology to a broader range of conditions; we leave this work for the future.
Overall, our results thus call for a deep overhaul of the prevailing theories of ice friction. The complex rheology of the interstitial material is shown to be a key ingredient, which has not been considered up to now in the theoretical frameworks describing water as a Newtonian fluid. Modeling the intertwinned relationships between the mechanical, rheological, and thermodynamic mechanisms is challenging but our results provide a guide and an experimental benchmark to revisit the standard framework of ice friction.

\section{CONCLUSION}

Thanks to our unique stroke-probe experimental setup, we have been able for the first time to bridge the gap between nanoscale and macroscale tribometry of ice, while fully characterizing the mechanics of the ice interface during frictional sliding. A key outcome of our study is the evidence for a complex mechanical behavior of the interstitial meltwater, which exhibits the rheology of a complex yielding material. Its large viscosity, coupled to an elastic response, yields an excellent hydrodynamic lubricant behavior, leading ultimately to low friction. Our experiments challenge the existing theories and should motivate a complete reformulation of the frameworks describing ice friction on the basis of the new fundamental insights unveiled here. Finally, the self-lubricating behavior of ice suggests to develop soft and phase-changing solids as antiwear tribofilms [46]. Ionic liquids, which have been shown to exhibit freezing in metallic confinement [47], are good candidates in this perspective.

\section{ACKNOWLEDGMENTS}

The authors are indebted to Martin Fourcade, Grégoire Deschamps, Yann Guigonnet, and Baptiste Claudon from the French biathlon team for many fruitful discussions on ski and waxing. The authors thank Axel Laborieux and Adrien Benusiglio for contributions at the early stages of the project, Joshua McGraw for his help with the silanization process, and Quentin Louis for his contribution to the experiments on polyethylene glycol. We also thank Annie Colin for discussions and help on the rheometry measurements. L. B. is indebted to Ludwik Leibler and Elisabeth Charlaix for many interesting discussions. The authors also thank Thomas Salez and Liliane Léger for their insight and feedback. We acknowledge support from ANR grant Neptune. A. S. acknowledges funding from the European Union's Horizon 2020 Framework Program/European Research Council Starting Grant No. 637748 NanoSOFT. L. B. acknowledges support from the European Union's Horizon 2020 Framework Program/European Research Council Advanced Grant No. 785911 Shadoks. 

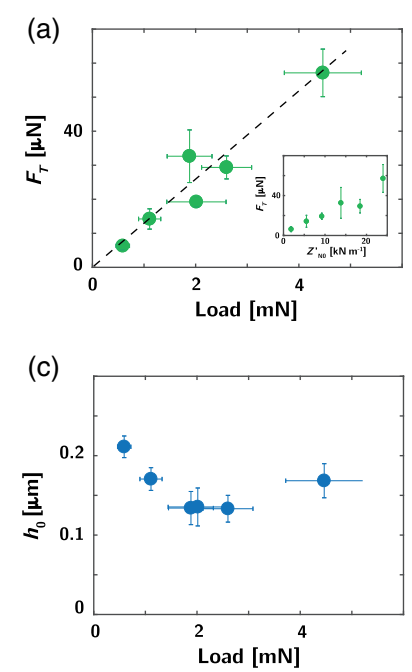

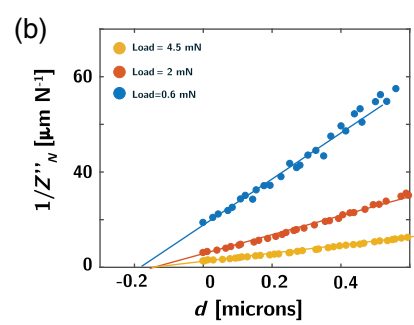

(d)

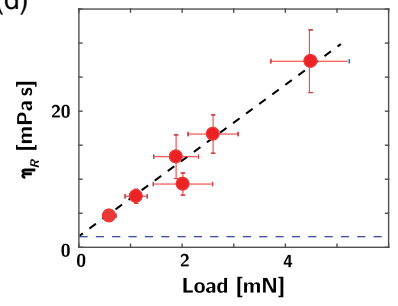

FIG. 6. Variations of the frictional and rheological properties of the interface with the prescribed load. (a) Friction evolution with the integrated load, highlighting a linear relation typical of a solidlike friction. (b) Retract curves for different regulating loads, showing a change in the slope rather than in the confinement distance with the regulating load. (c),(d) $h_{0}$ and $\eta_{R}$ as a function of the regulating load. The change in friction increase is shown to relate to the change in the local dissipative viscosity. The horizontal blue dashed line in (d) corresponds to the viscosity of supercooled water at $-6{ }^{\circ} \mathrm{C}$. The data correspond to measurements performed with a hydrophobic sphere.

\section{APPENDIX: INTERFACIAL WATER PROPERTIES}

\section{Dependence between the imposed load and the local properties of the interfacial medium}

In Fig. 6, we show how changing the regulating $Z_{N 0}^{\prime}$, hence the regulating load, affects the local interfacial properties. In the inset of Fig. 6(a), we show the variations of the frictional force as a function of the prescribed $Z_{N 0}^{\prime}$. We retrieve the load as described in Supplemental Material S2.2 and plot the variations of the friction force as a function of the prescribed load in the main panel of Fig. 6(a). We observe a linear variation reminiscent of a solidlike friction with an extremely small friction coefficient. In Fig. 6(b), we plot the inverse of the normal dissipative impedance during the retract phase for different prescribed loads. We observe that the horizontal intercept of the linear extrapolations is not strongly affected by changing the load, but the slope increases strongly. This shows that while the hydrodynamic thickness remains unchanged [Fig. 6(c)], the viscosity increases with the imposed load. We observe a linear dependence of the viscosity as function of the normal load [Fig. 6(d)].

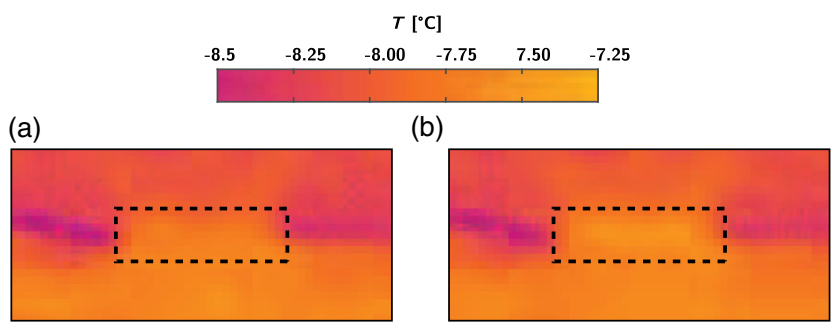

FIG. 7. Measurement of local heating during sliding. Measurements are obtained using an infrared camera Flir A600 series. The temperature scale is shown on right-hand axis. (a) At rest with $V=0$. (b) Under sliding conditions with $V=0.4 \mathrm{~m} \mathrm{~s}^{-1}$. Under sliding, we measure an average heating $\Delta T \approx 0.1^{\circ} \mathrm{C}$ over a zone of $\approx 300 \mu \mathrm{m}$ thickness (inside black dashed box).

\section{Local heating of the interface during sliding}

During sliding, we used an infrared camera to record the local heating of the bead and the underlying ice. As shown in Fig. 7, a very small increase in local temperature is observed. This is in agreement with recent measurements [27], reporting microscale infrared thermography and optical measurements of snow-grain contacts, which actually highlighted abrasion, rather than melting, of the interstitial contact region. This observation supports the hypothesis that the liquid film is not composed of pure meltwater at $0^{\circ} \mathrm{C}$, as assumed by standard frictional-melting models.

[1] B. Persson, Sliding Friction: Physical Principles and Applications, 2nd ed. (Springer-Verlag, Berlin, 2000).

[2] R. Rosenberg, Why Is Ice Slippery?, Phys. Today 58, No. 12, 50 (2005).

[3] T. H. Oosterkamp, T. Boudewijn, and J. M. J. van Leeuwen, Skating on Slippery Ice, Europhysics News 50, 28 (2019).

[4] K. Cuffey and W. S. B. Paterson, The Physics of Glaciers (Elsevier, New York, 2016).

[5] B. N. J. Persson, Ice Friction: Glacier Sliding on Hard Randomly Rough Bed Surface, J. Chem. Phys. 149, 234701 (2018).

[6] M. Faraday, XXIV. On Regelation, and on the Conservation of Force, Philos. Mag., Ser. 4 17, 162 (1859).

[7] A. Döppenschmidt and H. J. Butt, Measuring the Thickness of the Liquid-like Layer on Ice Surfaces with Atomic Force Microscopy, Langmuir, 16, 6709 (2000).

[8] H. Bluhm, F. D. Ogletree, C. S. Fadley, Z. Hussain, and M. Salmeron, The Premelting of Ice Studied with Photoelectron Spectroscopy, J. Phys. Condens. Matter 14, L227 (2002).

[9] M. E. Kornienko, N. L. Sheiko, O. M. Kornienko, and T. Y. Nikolaienko, Discrete Properties of Quasiliquid Water Film in the Ice Premelting Range. 1. Temperature Dependences of Water Nanofilm Thickness and Viscoelastic Properties of Polycrystalline Ice, Ukr. J. Phys. 58, 151 (2013).

[10] J.S. Wettlaufer, Ice Surfaces: Macroscopic Effects of Microscopic Structure, Phil. Trans. R. Soc. A 357, 3403 (1999). 
[11] M. M. Conde, C. Vega, and A. Patrykiejew, The Thickness of a Liquid Layer on the Free Surface of Ice as Obtained from Computer Simulation, J. Chem. Phys. 129, 014702 (2008).

[12] Y. Nagata, T. Hama, E. H. G. Backus, M. Mezger, D. Bonn, M. Bonn, and G. Sazaki, The Surface of Ice under Equilibrium and Nonequilibrium Conditions, Acc. Chem. Res. 52, 1006 (2019).

[13] B. Slater and A. Michaelides, Surface Premelting of Water Ice, Nat. Rev. Chem. 3, 172 (2019).

[14] M. A. Sánchez, T. Kling, T. Ishiyama, M. J. van Zadel, P. J. Bisson, M. Mezger, and M. J. Shultz, Experimental and Theoretical Evidence for Bilayer-by-Bilayer Surface Melting of Crystalline Ice, Proc. Natl. Acad. Sci. U.S.A. 114, 227 (2017).

[15] K. I. Murata, H. Asakawa, K. Nagashima, Y. Furukawa, and G. Sazaki, Thermodynamic Origin of Surface Melting on Ice Crystals, Proc. Natl. Acad. Sci. U.S.A. 113, E6741 (2016).

[16] B. N. J. Persson, Ice Friction: Role of Non-Uniform Frictional Heating and Ice Premelting, J. Chem. Phys. 143, 224701 (2015).

[17] F. P. Bowden and T. P. Hughes, The Mechanism of Sliding on Ice and Snow, Proc. R. Soc. A 172, 280 (1939).

[18] F. P. Bowden, Some Recent Experiments in Friction: Friction on Snow and Ice and the Development of Some FastRunning Skis, Nature (London) 176, 946 (1955).

[19] S. C. Colbeck, The Kinetic Friction of Snow, J. Glaciol. 34, 78 (1988).

[20] D. Buhl, M. Fauve, and H. Rhyner, The Kinetic Friction of Polyethylen on Snow: The Influence of the Snow Temperature and the Load, Cold Reg. Sci. Technol. 33, 133 (2001).

[21] L. Baürle, D. Szabò, M. Fauve, H. Rhyner, and N. D. Spencer, Sliding Friction of Polyethylene on Ice: Tribometer Measurements, Tribol. Lett. 24, 77 (2006).

[22] A. M. Kietzig, S. G. Hatzikiriakos, and P. Englezos, Ice Friction: The Effects of Surface Roughness, Structure and Hydrophobicity, J. Appl. Phys. 106, 024303 (2009).

[23] A. M. Kietzig, S. G. Hatzikiriakos, and P. Englezos, Physics of Ice Friction, J. Appl. Phys. 107, 081101 (2010).

[24] D. C. B. Evans, J. F. Nye, and K. J. Cheeseman, The Kinetic Friction of Ice, Proc. R. Soc. A 347, 493 (1976).

[25] W. Ambach and B. Mayr, Ski Gliding and Water Film, Cold Reg. Sci. Technol. 5, 59 (1981).

[26] H. Strausky, J. R. Krenn, A. Leitner, and F. R. Aussenegg, Sliding Plastics on Ice: Fluorescence Spectroscopic Studies on Interfacial Water Layers in the $\mu \mathrm{m}$ Thickness Regime, Appl. Phys. B 66, 599 (1998).

[27] J. H. Lever, S. Taylor, A. J. Song, Z. R. Courville, R. Lieblappen, and J.C. Weale, The Mechanics of Snow Friction as Revealed by Micro-Scale Interface Observations, J. Glaciol. 64, 27 (2018).

[28] L. Canale, A. Laborieux, A. A. Mogane, L. Jubin, J. Comtet, A. Lainé, L. Bocquet, A. Siria, and A. Niguès, MicroMegascope, Nanotechnology, 29, 355501 (2018).

[29] A. Lainé, L. Jubin, L. Canale, L. Bocquet, A. Siria, S. H. Donaldson, Jr., and A. Niguès, MicroMegascope Based Dynamic Surface Force Apparatus, Nanotechnology 30, 195502 (2019).

[30] N. T. Garabedian, H. S. Khare, R. W. Carpick, and D. L. Burris, AFM at the Macroscale: Methods to Fabricate and
Calibrate Probes for Millinewton Force Measurements, Tribol. Lett. 67, 21 (2019).

[31] J. Comtet, G. Chatté, A. Niguès, L. Bocquet, A. Siria, and A. Colin, Pairwise Frictional Profile between Particles Determines Discontinuous Shear Thickening Transition in Non-Colloidal Suspensions, Nat. Commun. 8, 15633 (2017).

[32] See Supplemental Material at http://link.aps.org/ supplemental/10.1103/PhysRevX.9.041025 for details on the experimental setup, procedure, and control experiments.

[33] J. Vermant, P. Moldenaers, J. Mewis, M. Ellis, and R. Garritano, Orthogonal Superposition Measurements Using a Rheometer Equipped with a Force Rebalanced Transducer, Rev. Sci. Instrum. 68, 4090 (1997).

[34] H. Bluhm, T. Inoue, and M. Salmeron, Friction of Ice Measured Using Lateral Force Microscopy, Phys. Rev. B 61, 7760. (2000).

[35] B. Weber, Y. Nagata, S. Ketzetzi, F. Tang, W. Smit, H. Bakker, E. Backus, M. Bonn, and D. Bonn, Molecular Insight into the Slipperiness of Ice, J. Phys. Chem. Lett. 9, 2838 (2018).

[36] D. Ortiz-Young, H. C. Chiu, S. Kim, K. Voïtchovsky, and E. Riedo, The Interplay between Apparent Viscosity and Wettability in Nanoconfined Water, Nat. Commun. 4, 2482. (2013).

[37] L. Bocquet and E. Charlaix, Nanofluidics, from Bulk to Interfaces, Chem. Soc. Rev. 39, 1073 (2010).

[38] R. G. Larson, The Structure and Rheology of Complex Fluids (Oxford University Press, New York, 1999).

[39] B. Cross, C. Barraud, C. Picard, L. Léger, F. Restagno, and E. Charlaix, Wall Slip of Complex Fluids: Interfacial Friction versus Slip Length, Phys. Rev. Fluids 3, 062001(R) (2018).

[40] J. Hallett, The Temperature Dependence of the Viscosity of Supercooled Water, Proc. Phys. Soc. 82, 1046 (1963).

[41] A. Cuenca and H. Bodiguel, Submicron Flow of Polymer Solutions: Slippage Reduction due to Confinement, Phys. Rev. Lett. 110, 108304 (2013).

[42] L. Bureau, T. Baumberger, and C. Caroli, Rheological Aging and Rejuvenation in Solid Friction Contacts, Eur. Phys. J. E 8, 331 (2002).

[43] K. Nichol and M. van Hecke, Flow-Induced Agitations Create a Granular Fluid: Effective Viscosity and Fluctuations, Phys. Rev. E 85, 061309 (2012).

[44] S. K. Lee, R. Han, E. J. Kim, G. Y. Jeong, H. Khim, and T. Hirose, Quasi-Equilibrium Melting of Quartzite upon Extreme Friction, Nat. Geosci. 10, 436 (2017).

[45] F. Boyer, E. Guazzelli, and O. Pouliquen, Unifying Suspension and Granular Rheology, Phys. Rev. Lett. 107, 188301 (2011).

[46] N. N. Gosvami, J. A. Bares, F. Mangolini, A. R. Konicek, D. G. Yablon, and R. W. Carpick, Mechanisms of Antiwear Tribofilm Growth Revealed In Situ by Single-Asperity Sliding Contacts, Science 348, 102 (2015).

[47] J. Comtet, A. Niguès, V. Kaiser, B. Coasne, L. Bocquet, and A. Siria, Nanoscale Capillary Freezing of Ionic Liquids Confined between Metallic Interfaces and the Role of Electronic Screening, Nat. Mater. 16, 634 (2017). 